Matthew Davis*, Michael Proctor and Buder Shageer

\title{
A Systems-Of-Systems Conceptual Model and Live Virtual Constructive Simulation Framework for Improved Nuclear Disaster Emergency Preparedness, Response, and Mitigation
}

DOI 10.1515/jhsem-2015-0051

Abstract: Nuclear disasters have severe and far-reaching consequences. Emergency managers and first responders from utility owners to local, state, and federal civil authorities and the Department of Defense (DoD) must be well prepared in order to rapidly mitigate the disaster and protect the public and environment from spreading damage. Given the high risks, modeling and simulation (M\&S) plays a significant role in planning and training for the spectrum of derivate scenarios. Existing reactor models are largely legacy, stove-piped designs lacking interoperability between themselves and other M\&S tools for emergency preparedness system evaluation and training. Unmanned systems present a growing area of technology promising significant improvement in response and mitigation. To bridge the gap between current and future models, we propose a conceptual model (CM) for integrating live, virtual, and constructive (LVC) models with nuclear disaster and mitigation models utilizing a system-ofsystems (SoS) approach. The CM offers to synergistically enhance current reactor and dispersion simulations with intervening avatar and agent simulations. The SoS approach advances life cycle stages including concept exploration, system design, engineering, training, and mission rehearsal. Component subsystems of the $\mathrm{CM}$ are described along with an explanation of input/output requirements. A notional implementation is described. Finally, applications to analysis and training, an evaluation of the CM based on recently proposed criteria found in the literature, and suggestions for future research are discussed.

*Corresponding author: Matthew Davis, University of Central Florida - Industrial Engineering and Management Systems, Orlando, FL, USA, e-mail: matt.davis@knights.ucf.edu Michael Proctor and Buder Shageer: University of Central Florida - Industrial Engineering and Management Systems, Orlando, FL, USA 
Keywords: analysis; incident management; interoperability; nuclear emergency planning; simulation; system of systems; systems engineering; training; unmanned system.

\section{Introduction}

Nuclear power generating facilities are designed with extensive redundant safety measures. While the probability of a nuclear disaster is extremely low, rare events may quickly lead to a disaster with the scale of Fukushima or even Chernobyl (OPA 2013a,b; TEPCO 2013; NRC 2014a,b; DOE 2015). One hundred thirty five domestic nuclear reactors (NRC 2015b) and over 500 nuclear reactors operating or under construction worldwide (WNA 2015) multiplies the possibility of a widespread disaster - whether originating from human error, design flaw, external earthquake, tsunami, hurricane storm surge, et cetera. Despite the extensive onsite nuclear facility safety systems and personnel training, efforts to mitigate the effects of a rare event disaster may extend offsite to the utility company, pooled utility response centers, local, state, and federal government agencies, military organizations such as the Joint Task Force Civil Support (JTF-CS) (JTFCS 2015b) and resources from other nations. Given the high risks of radiation to human life, modeling and simulation (M\&S) may play a significant role in planning and training of offsite responders to potential disaster scenarios. Complicating preparedness of offsite responders is the lack of interoperability between legacy, stove-pipe nuclear reactor simulations and live, virtual, and constructive (LVC) simulations often used in planning and training. To bridge the lack of interoperability, we propose a system-of-systems (SoS) conceptual model (CM) for integrating LVC simulations into a framework containing nuclear reactor and radiation dispersion models.

The three most publicized and possibly worst nuclear power plant disasters are Three Mile Island, Chernobyl and Fukushima Dai-ichi. Chernobyl nuclear disaster breached site containment and spread radiation contaminants across Europe (OPA 2013a; UNSCEAR 2000). Fukushima Dai-ichi nuclear plant design and safety operations addressed likely earthquakes and tsunamis. Despite these site precautions, the unprecedented March 11, 2011 earthquake created a chain of events that resulted in a rare nuclear disaster. The initial earthquake left the reactors undamaged but damaged offsite power delivery systems. The damaged offsite power systems caused the entire plant to transfer over to onsite diesel generators. Fifty min later the tsunami struck. The height of the tsunami overtopped the seawall, and flooded the generators and the onsite backup batteries. With the 
total loss of power, the inability to monitor and maintain cooling eventually led to partial core meltdowns in reactors 1-3(TEPCO 2013). With this series of rare events, the Fukushima Dai-ichi nuclear disaster breached local defenses and spread radiation contaminants in the air across a large area of Japan (UNSCEAR 2014) and spread radiation contaminants through Pacific ocean currents (UNSCEAR 2014) reaching measurable radiation levels in the western United States (EPA 2011). Subsequent to the disaster the United States Nuclear Regulatory Commission (NRC) issued "Recommendations for Enhancing Reactor Safety in the 21st Century: The Near-Term Task Force Review of Insights from the Fukushima Daiichi Accident" and reiterated their Defense-in-Depth philosophy. Defense-inDepth weaves together onsite and off-site responders and offers "protection from design-basis natural phenomena, mitigation of the consequences of accidents, and EP [Emergency Preparedness]" (Miller et al. 2011).

Offsite manned helicopters are popular choices for disaster response and mitigation efforts due to their flexibility and responsiveness. At Three Mile Island helicopters sampled atmospheric radioactivity above the plant within hours after the event began (OPA 2013b). At Chernobyl Soviet military helicopters dumped, at times by hand, sand and boron directly onto the fire, molten core, and contaminated reactor debris (OPA 2013a). At Fukushima, Japan Self-Defense Force (JDSF) helicopters and crews attempted to stop core meltdown by dumping fresh water into evaporating spent fuel rod cooling pools (NRC 2012).

In each of these passed cases manned helicopters put the aircrews at significant risk. At Three Mile Island, radiation levels exposed helicopters and crews contracted by the utility company to unknown risk during sampling missions. At Chernobyl, helicopter crews from the Soviet military passed through the highly radioactive plume of super-heated gas rising from the fire resulting in many deaths (Johnson 2006). Additionally, four crewmembers of a Soviet helicopter were killed when their aircraft struck crane cables near the reactor and fell from the sky (Pripyat.com 2006). At Fukushima Dai-ichi, JDSF helicopter response and mitigation efforts were quickly abandoned out of fear of aircrew radiation exposure. Illustrating the succession of response from utility through civil authorities to defense resources and importance of defense in depth, helicopters in two of these three incidents, Chernobyl and Fukushima, were owned and operated by the militaries of their respective nations and in the third the utility owner contracted the helicopter (OPA 2013b).

Given the increasing need to improve crisis management processes (Deverell 2012) and the costly and far reaching consequences of potential future nuclear reactor disasters, governing agencies must investigate innovative strategies or alternative technologies to respond to crises and limit the scale of subsequent damage. To achieve the President's National Preparedness Goal (DHS 2015), 
utility, local, state, federal, and military first responders must plan and train with these new technologies to rapidly and effectively integrate them into the five mission areas of emergency management within the National Preparedness System (FEMA 2014) as shown in Figure 1. "Modeling and simulation techniques can help address many of the challenges brought forth by the need for incident management" and "their usefulness increases exponentially with incident size" (Jain and McLean 2006). As reported by Van Niekerk et al. (2015), the application of simulation to emergency management exercises is well established in the literature. Further, simulation-based acquisition (Zittel 2001; Proctor et al. 2003) and system design and engineering (Brantley et al. 2002) may enable safe and cost effective exploration of the contribution and possible acquisition of these new unmanned technologies for nuclear disaster response, mitigation, and recovery.

In order to leverage M\&S to achieve NRC goals, a CM of the mission space is necessary. A CM is the basis for modeling behavior of individual components and their interactions within a federation. A "federation" is a group of simulations each with the ability to operate independently but interoperate concurrently to accomplish missions. A federate is a simulation within the federation. Concurrent interoperation requires effective communications. Mutually agreed upon federation common language, communications network and related protocols enable communications and concurrent interoperation. Protocols or "systems of rules for governance" encompass language, hardware, and software standards used to form and transmit data between the models, simulations, and systems participating in the simulation scenario. Specificity of meaning and context of language may be explicitly or implicitly governed by protocols but applied within the context of the federation scope. The scope of the federation is determined by the scope of shared purpose agreed to by the federates.

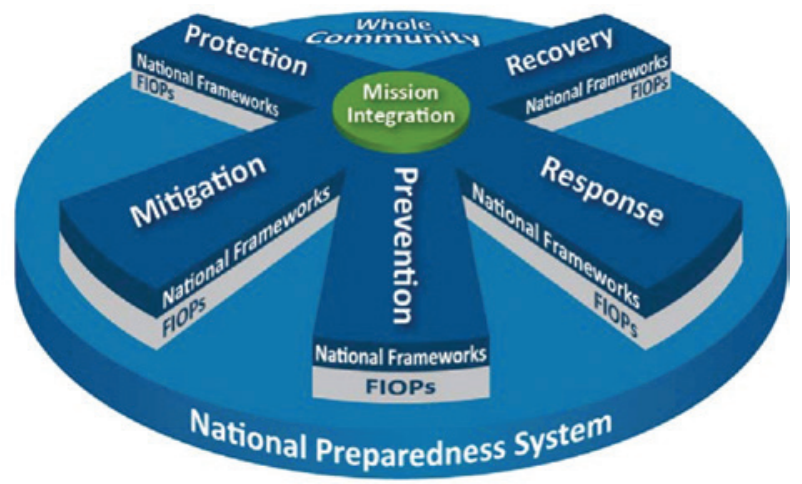

Figure 1: Mission Integration within the National Preparedness System (FEMA 2014). 


\section{Unmanned Systems and the Impact on Nuclear Disaster M\&S}

Unmanned systems, M\&S, and LVC simulation federations are well known to nuclear disaster emergency managers and response planners.

In future disasters use of technology-based unmanned systems instead of manned systems may eliminate aircrew risks, expand the operational envelope of the aircraft increasing the potential for mission success, support many nuclear power generating facilities from one location, extend mission time and increase resilience to radioactive, high heat environments when compared to existing manned solutions. Unlike humans, unmanned systems may be dispensable. Unmanned aerial vehicles "(UAVs) are used for missions that are 'dull, dirty, and dangerous"” presenting "a high risk factor for pilots” (van Blyenburgh 2000). At Chernobyl remotely operated vehicles removed highly radioactive debris from a rooftop (Johnson 2006). Currently Fukushima Dai-ichi, Tokyo Electric Power Company (TEPCO) uses a variety of ground and submersible systems to assist in recovery within the reactor buildings (Oikawa 2015). UAVs are rapidly being adopted through systems engineering for disaster response agencies (Tomaszewski et al. 2015). Engineering and technology advances in unmanned system now allow rotary-wing missions previously manned to be accomplished using rotary-wing unmanned aerial systems (RUAS) (Saggiani and Teodorani 2004; Farradyne 2005; UTM 2007; Bernard et al. 2008; McGonigle et al. 2008; Alexis et al. 2009; Marconi et al. 2012; Mase 2013). Unmanned systems include aerial systems, submersible unmanned systems (Oikawa 2015), maritime unmanned surface vehicle (USV) technology (Campbell et al. 2014; Sharma et al. 2014; Svec et al. 2014) and ground based systems that may "walk" like a person or animal (Hsu 11/2014; Oikawa 2015). Given the proximity of many nuclear reactor sites to large bodies of water, the implementation of USVs in disaster response planning is a natural fit. Notionally the employment of new technologies offer potential new opportunities to not just recover from a nuclear event but respond, mitigate effects and protect the surrounding area by containing the scale and scope of a nuclear reactor disaster.

M\&S is widely used within NRC partnering institutions. NRC in partnership with Sandia National Laboratory maintains several dispersion, dosing, and severe accident reactor models. These models are all specialized to produce high fidelity predictions of very specific components of fuel and reactor behavior, accident progression, radionuclide transport, and dosing and some do so with fairly fast run times (NRC 2015a). Literature on operations research, nuclear power plants, nuclear reactor accidents and simulation is vast with (Saggiani and Teodorani 
2004; Farradyne 2005; Bernard et al. 2008; McGonigle et al. 2008; Peräjärvi et al. 2008; Alexis et al. 2009; Flint et al. 2009; Girault et al. 2010; Ianovsky and Kreimer 2011; Alver et al. 2012; Marconi et al. 2012; Towler et al. 2012; Ai-Omari et al. 2013; Chaimatanan et al. 2013; Mase 2013, 2015; Holden and Dickerson 2013; Liu et al. 2014; MacFarlane et al. 2014; Ouyang et al. 2014; Hu et al. 2015; Sheng et al. 2015; Wang et al. 2015b) being but a few examples. Many of these works utilize simulation techniques, due in part to the complex nature of UAS and their environmental interactions and the difficulty in analytically evaluating real-world systems (Law and Kelton 2000). A generalized framework for simulation based analysis exists for UAS (Perhinschi et al. 2010) and unmanned systems (Flint et al. 2009; Liu et al. 2014). Beyond those maintained by NRC and Sandia, M\&S are available for both reactor thermohydraulics modeling (Wang et al. 2005; Wang et al. 2015b) and material dispersion modeling. Also outside NRC, M\&S has a long history in DoD that may support nuclear disaster mitigation scenarios (Hollenbach and Alexander 1997). Hazard Prediction and Assessment Capability (HPAC) is a widely used tool (Chang et al. 2005; Platt et al. 2014; Hanna and Chang 2015; Singh et al. 2015) licensed by the Defense Threat Reduction Agency. HPAC "assists in emergency response to hazardous agent releases. Its fast running, physics-based algorithms enable users to model and predict hazard areas and human collateral effects in minutes. HPAC provides the capability to accurately predict the effects of HAZMAT [Hazardous Material] releases into the atmosphere and their impact on civilian and military populations" (DoD 2013). Heffelfinger et al. (2013) provide a thorough treatment of the military role in domestic Chemical, Biological, Radiological, Nuclear, and High Explosive (CBRNE) incident response. "Bringing together the myriad of capabilities of the military forces in a seamless response is a daunting task." Realistic training is integral to successful CBRNE response and simulation federations plays a key role in this training (Heffelfinger et al. 2013).

LVC simulation federations are widely used by the military for such training (Hodson and Hill 2014) using several different interoperability protocols. Example interoperability protocols include: distributed interactive simulation (DIS), high level architecture (HLA), and test and training enabling architecture (TENA). In addition to training and mission rehearsal, the resultant simulation federation may support concept development, analysis, test and evaluation, and system development life cycle phases. As in past system developments, M\&S will be key to successful integration of unmanned systems into nuclear disaster response plans (INNG 2015).

Simulation federations that closely parallel area-wide nuclear disaster scenarios possibly faced by emergency response personnel include the US Army Dugway Proving Ground's Chemical-Biological Simulation Suite (CBSS). CBSS “is 
a set of distributed simulation software tools designed to represent all aspects of CB [Chemical Biological] defense on the tactical battlefield." It computes transport and dispersion using high fidelity vapor, terrain, and weather models and delivers output to other federates via its Chemical/Biological Synthetic Natural Environment (Baker 2012). The CBSS is used to:

- Develop effective CB defense materiel

- Evaluate tactics, techniques, and procedures (TTP)

- Provide constructive testing over a wide range of terrain, weather, and delivery conditions

- Provide broad scenario-based training

- Support live sensor testing at Dugway (Baker 2012)

Another DoD example is the Naval Air Warfare Center Training Systems Division's (NAWCTSD) Chemical Biological Radiological Nuclear Explosive Tactical Training System that focuses on maintaining situational awareness of ground forces within a CBRNE environment (NAWCTSD 2014). There are also other dispersion models at various levels of fidelity and interoperability (Hill 2003).

Figure 2 illustrates the current state of simulation interoperability. As indicated by the dashed lines, there are very limited existing capability to integrate data from and provide input to current dispersion and reactor models from broader, large audience, LVC federations (Lee 2002; WSC 2012; Baker 2012; NAWCTSD 2014). Future designs require more complex, responsive, and interoperable solutions to support future planning and training for disaster response, especially as the use of modern avatars and agents grows.

\section{System of Systems Conceptual Model for Improved Nuclear Disaster Emergency Preparedness, Response and Mitigation}

The 2011 DHS/NIST Workshop on Homeland Security Modeling \& Simulation identified as needing to be addressed were "use of a system-of-systems (SoS) engineering approach to the development of applications" and "integration of hazardous material release models and simulations with other simulation and training applications, e.g. incident management systems" (Sugiyama 2011). Hodson and Hill (2014), "[Conceptual modeling] is almost certainly the most important aspect of a simulation project" and is widely discussed in the literature (Hamilton 2006; Özhan et al. 2008; Robinson 2008a,b; Robinson 2010; Gaffney and Vincent 2011; Graniela and Proctor 2012; Sokolowski et al. 2012; 


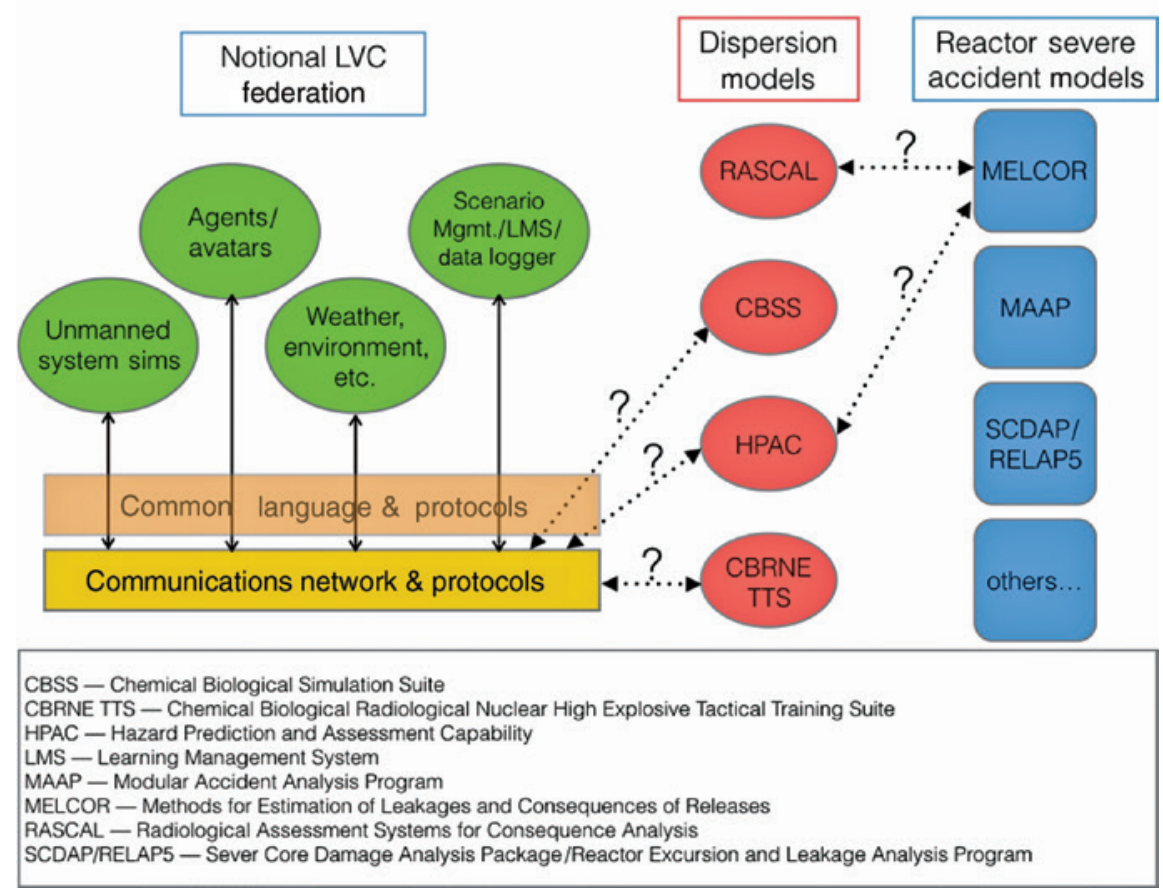

Figure 2: Current Level of Simulation Interoperability Among Potential Nuclear Disaster Emergency Preparedness, Response and Mitigation Federates.

Çelik et al. 2013; Hodson et al. 2014; Morris et al. 2014; Ünal and Topçu 2014). Our CM approach incorporates legacy models and the notional federation shown in Figure 2 within the context of SoS. Maier describes a SoS as: (1) Components have valid, fulfillable purposes independent of the larger system, and (2) components are managed in view of their own objectives rather than the objectives of the larger system (Maier 1998). Rainey and Tolk distinguish SoS as: "SoS are distinguished from other systems by formation from independently operated and managed components" (Jones 2015). A SoS improves management, planning, response, and mitigation in a fashion similar to other recent approaches (Liang et al. 2015; Stephens et al. 2015). A SoS approach elicits aggregate system behavior known as emergence (Fisher 2006). Emergent behavior may have beneficial or detrimental effects (Jones 2015). Emergent behavior enables a clearer understanding of the true utility of unmanned systems within nuclear disaster response.

The flexibility offered by a SoS CM approach is especially pertinent given the findings of Adalja et al. (2015), who report incident managers find current exercises "unrealistic" or "antiquated". 
A notional CM integrating unmanned systems into nuclear disaster emergency management planning is shown in Figure 3. The creation of a CM includes identification of an experimental frame where the importance and relationships of basic components are identified from a SoS perspective (Jones 2015). The expanse of tasks to which unmanned systems may be applied in future nuclear disasters and spectrum of alternative technologies is yet to be determined. To limit research scope, this article focuses on the manned helicopter water delivery mission at Fukushima as a notional illustration of unmanned systems employment during nuclear disaster response. While other tasks may exist, the Fukushima Dai-ichi actual helicopter tasking evolved avoiding a nuclear meltdown by maintaining sufficient water levels in the spent fuel pool (SFP) of Reactor 4. A thorough treatment of the timeline and situation evolution is available from several sources (Miller et al. 2011; ANS 2012; WNA 2012; TEPCO 2013). As a representative case for interoperable M\&S, the task at Reactor 4 was to deliver helicopter slung load water from above to the exposed SFP.

\section{Component Analysis of the Conceptual Model}

This section expands on components highlighted in Figure 3 from a SoS perspective as applied to the SFP scenario.

Framing the real world problem as a SoS where many complex subsystems interoperate within the larger system leads naturally to a conceptualization of

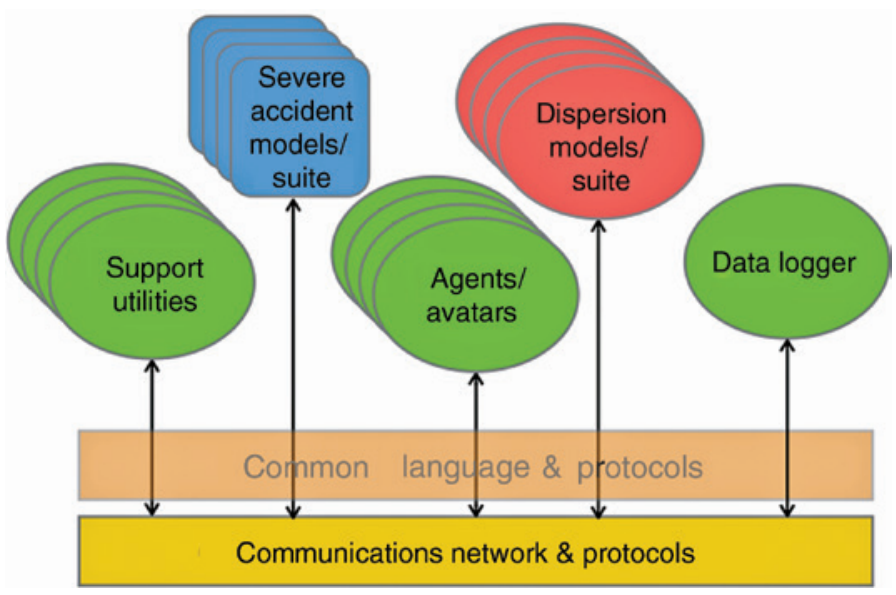

Figure 3: Notional Conceptual Model. 
the problem in simulation as a composition of interoperating models, each of which simulates the behavior of smaller subsystems of the overarching system. Currently replicating the Fukushima Dai-ichi scenario using existing models and simulations would involve extensive and time consuming hand coding to achieve interoperation.

For the purpose of SoS component design given the SFP scenario as representative of any number of missions, the SFP is assumed to be in a radiated environment and potentially a source of radiation itself. The general SFP mission is to replenish and then maintain water level and temperature within the SFP above and below (respectively) NUREG-1738 thresholds. These thresholds are related to the depth of the water above the top of the fuel rods housed in the SFP and the water temperature's associated evaporation/boil rate. In our example, mission success occurs when the water level and temperature in the SFP are within operating parameters of normal operations. Mission failure occurs when outside threshold boundaries. NUREG-1738 states "The end state ... was an SFP water level 3 feet above the top of the fuel. This simplified end state was used because recovery below this level, given failure to recover before reaching this level, was judged to be unlikely given the significant radiation field in and around the SFP at lowered water levels" (Collins and Hubbard 2001). Maintaining SFP temperatures via various water delivery methods whether by way of RUAS or other unmanned systems may be representative of a SoS in future nuclear disaster scenario. M\&S of these scenarios would illuminate issues and possible solutions arising from this very complex task within a dangerous nuclear environment with multiple factors affecting the effectiveness and efficiency of any responding unmanned systems deployed to mitigate or recover from the disaster.

Components within the M\&S of a nuclear disaster and referent sub-systems include:

- The reactor and SFP thermohydraulics

- The RUAS or unmanned system of interest within a chosen scenario

- Agents including the ground control station and maintenance/refuel site for the RUAS including the fresh water source

- Radionuclide dispersion to the environment

- Atmospherics - weather, wind, etc. at and around the reactor site

In addition to these real-world systems that interact in the SoS, there are additional sub-systems that should be accounted for in the simulated SoS:

- A communication control module - interoperability standard ensuring module-to-module communication (DIS protocol, HLA RTI, TENA, etc.)

- A scenario management module

- A data logging module 
Following the development process developed by Zeigler et al. (2000) and outlined by Rainey and Tolk (Jones 2015), the CM below can be used to develop a software specific instance of a simulation model. Each of these is represented in an expanded view of the CM in Figure 4 and explained in detail in the following paragraphs.

Dynamic Terrain Services - Considering each module from left to right, traditionally, terrain begins as a static environment selected by Federation members as an area of interest. United States Geological Survey provides a source of validated elevation post, surface imagery, and terrain surface feature data through its openly accessible Earth Explorer site (USGS 2016). Advances in computing capability enable terrain that influences agents and avatar ability to accurately navigate and progress across different terrain types (Graniela and Proctor 2012), generate in real time physics-based terrain modifications due to underlying disruptive impulses (Rami and Proctor 2007), and implement dynamic terrain techniques in large scale exercises (Ellis et al. 2010). The disruption of movement of ground vehicles justifies an independent representation within the illustrated CM as seen in Figure 4.

RUAS Avatar - RUAS may avoid issues such as flooding, roadway or bridge collapse, and obstacles that confront unmanned ground systems. RUAS may be represented in a simulation as a pre-scripted agent or as a human operator avatar. Proctor and Paulo (1996) established as far back as 1996 that agent representation in synthetic environments often operate significantly different than live opera-

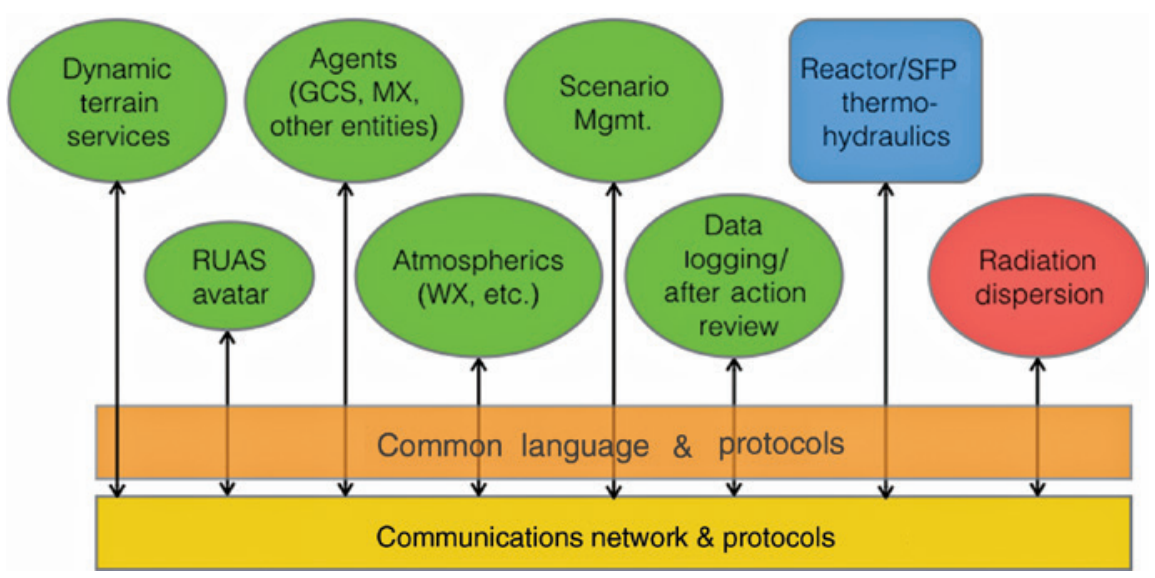

Figure 4: Components of the Conceptual Model. 
tors in the real environments. Avatars facilitate live humans remotely operating unmanned systems in a simulation thus avoiding many anomalies generated by pre-scripted agents. Avatars require a human interface comparable to the real world interface. Further, in RUAS avatar scenario expected research and training challenges of interest also include team situational awareness (Proctor et al. 2004), field of view of individual pilot/co-pilot (Covelli et al. 2010), and unique to this research, flight crew controlling slings, lifts, cranes, and scoops. The RUAS would also need to include shielding of electronics and sensors from radiation and heat.

Agents - Most entities, while important, may not be central to the focus of a scenario and can therefore be represented by agents with varying levels of artificial intelligence. Notionally, a model within the RUAS scenario could represent the RUAS's Ground Control Station and its operators as well as the maintenance and refuel facilities and personnel. All the other personnel and activities involved in the response and mitigation efforts are represented here as well. If an RUAS agent is to be created, consideration is be given not only to the obvious need for accuracy of the internal logic of the agent, but "correlated" sensor models that "evolve with... radiant energy, environmental effects, and sensor technology" across the electromagnetic spectrum with a simulation federation (Proctor and Connors 2000).

Atmospherics - Atmospherics is a key component in determining radionuclide dispersion, thus a separate module is dedicated to handling its accurate representation (Platt et al. 2014; Singh et al. 2015; Hanna and Chang 2015). Atmospherics, particularly turbulence, may adversely impact helicopter pilot performance with increased weight with respect to the velocity and altitude parameters of the helicopter (Proctor et al. 2007). Robust analytical and training capability necessitates this module offer users various weather generation techniques, so user definition of weather is another input stream.

Scenario Management - In order for analysis or training to occur, initial conditions must be set, operating parameters established, and an end state identified. These parameters are notionally bounded by the experimental frame and derived from the experimental design in the case of analysis and from the training objectives in the case of training. While these parameters are initially expressed in data formats oriented to human understanding (tables, figures, plain text), they must be delivered to the simulated SoS in a machine digestible format (Ünal and Topçu 2014; Holden and Dickerson 2013). This module monitors the simulation during execution to ensure all participating sub-systems, or federates, are abiding by the established operating parameters. 
Data Logging - To achieve the end goal of optimizing RUAS implementation or evaluating trainee performance, system behavior must be tracked and logged for post-scenario evaluation. This module is similar to after action review tools present in many DoD training simulations (Meliza et al. 2007; Green et al. 2011; Sawyer and Deering 2013) and such a tool could notionally be applied to an implementation of our CM to accomplish this task. Data Logging facilitates circumspect analysis and effective post-training debrief.

Reactor and SFP Thermohydraulics - This module is representative of a high fidelity model of the nuclear physics and thermodynamics taking place in and around the reactor and the SFP. The actual nuclear reactions as well as the heat transfer processes taking place in many areas near the reactor including within the SFP where our scenario is focused are highly complex and critical to accurate analysis of unmanned systems capability to mitigate source term release. Therefore, a model specifically built for predicting severe accident behavior is highly desirable. Several alternatives exist to include MELCOR, SCDAP/RELAP5, and MAAP (Wang et al. 2005; Polo-Labarrios and Espinosa-Paredes 2015).

Dispersion - High fidelity representation of dispersion of radionuclides to the surrounding environment is the primary concern with any nuclear disaster. Alternatives for dispersion and dose rates for personnel operating within affected areas as well as radionuclide deposit on structures and vehicles traveling through affected areas include HPAC, RASCAL, ALOHA, AUSTOX, AUSPLUME, and CBSS (Hill 2003; Baker 2012; NRC 2015a).

\section{Common Language, Communications Network and Related Protocols -}

Federation agreed upon common language, communications network and related protocols are widely used by M\&S communities to achieve the desired level of interoperability between simulation systems. DIS, HLA, and TENA are three such standards, and detailed descriptions of each are available from several sources (IEEE 2010, 2012; Jones 2015; TRMC 2015). Interoperability and composability are foundations to M\&S over the last 15 years (Kasputis and Ng 2000; Davis and Anderson 2004; Yilmaz 2004; Davis and Tolk 2007). Interoperability is critical to proper SoS function (Jones 2015) but interoperability is a spectrum and enhanced SoS performance comes as the degree of interoperability between the sub-systems increases (Tolk and Muguira 2003).

Table 1 below summarizes component purpose and sample input/output message requirements. Descriptions of input/output message requirements indicate the general data flow from component to component. Detailed content of message traffic is far too great to describe herein. 


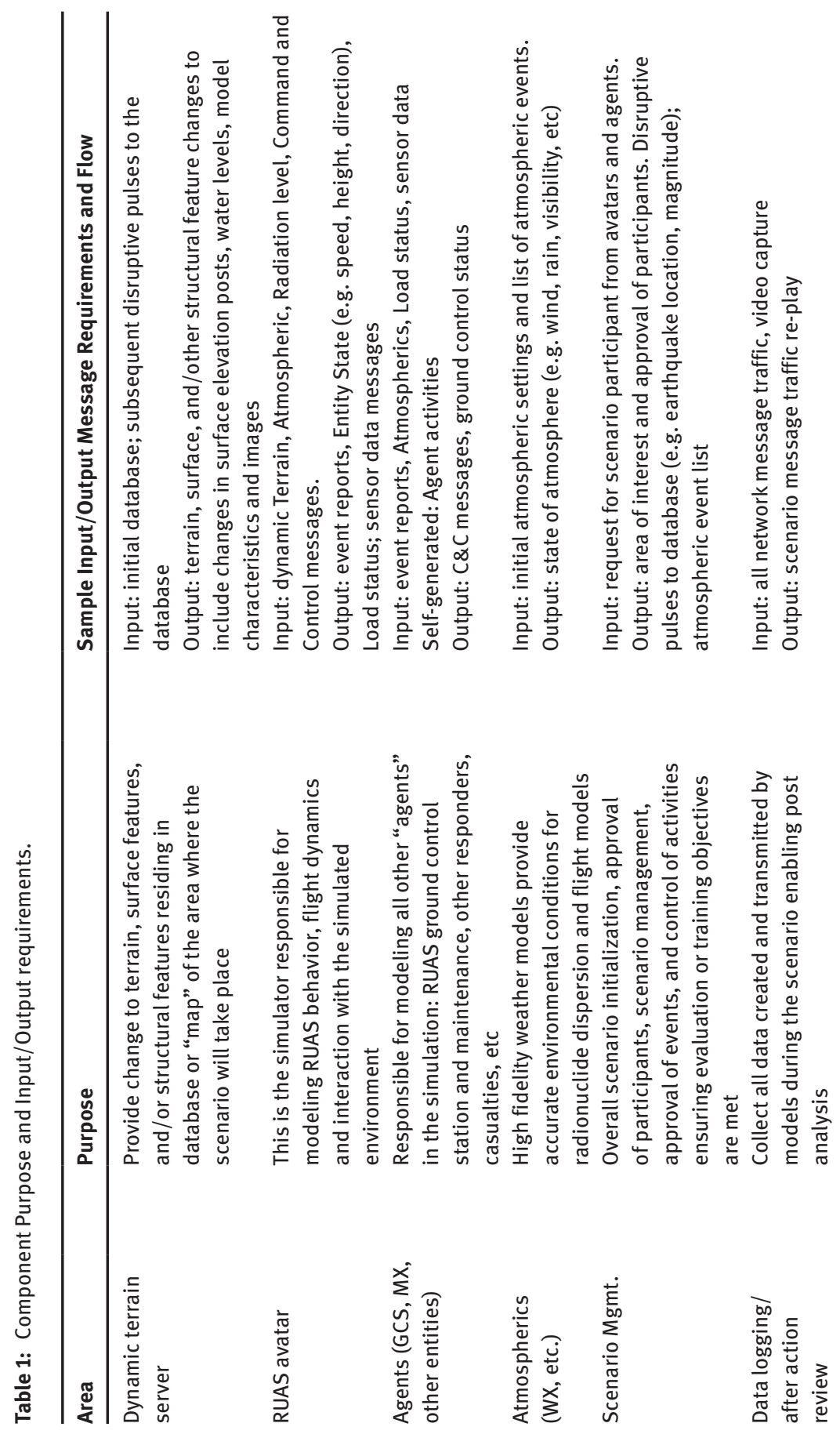




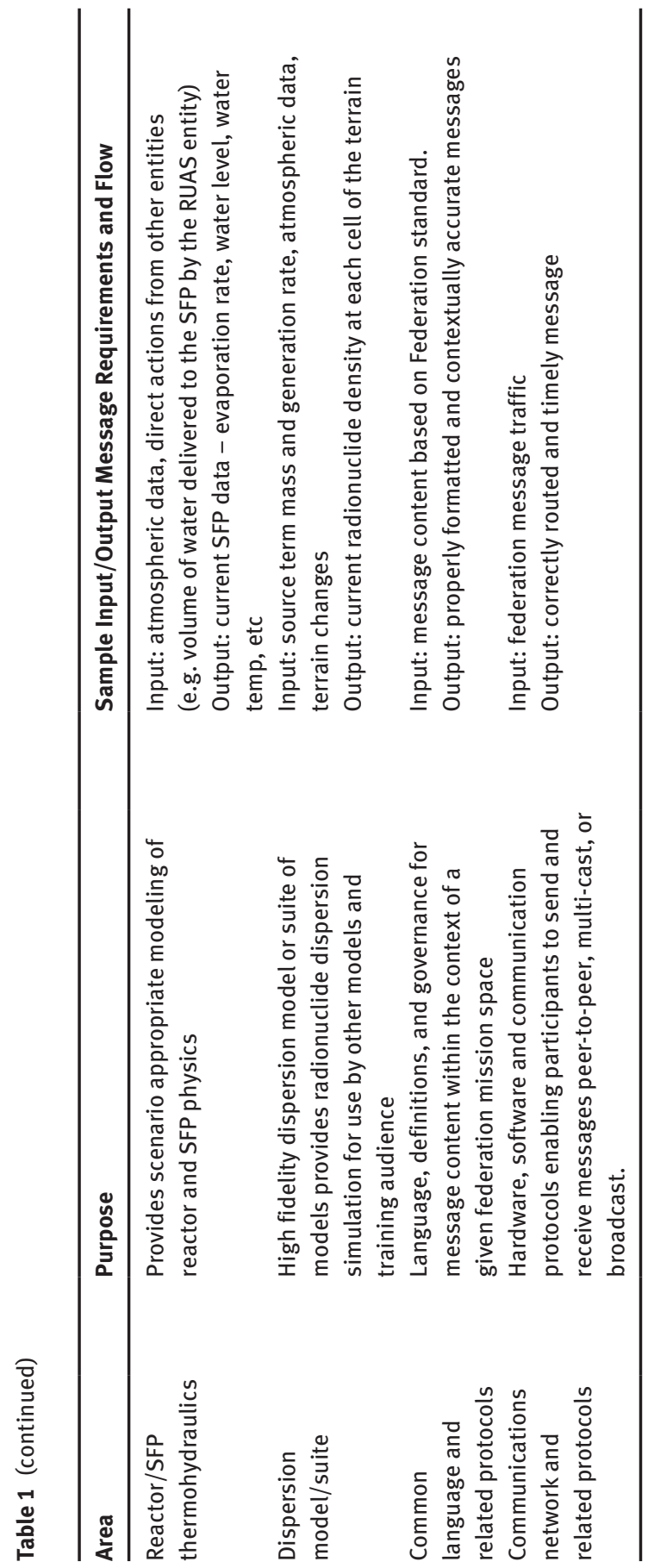




\section{LVC Simulation Framework}

Identifying simulation tools or suites able to support the CM in Figure 4 is a major task of a federation. Tailoring a simulation federation that addresses various organizational and agency needs generated by utility operator, local and state government, NRC, and FEMA requirements to conduct evaluated exercises every 2 years (NRC 2011) may likely involve writing software interfaces. Figure 5 below illustrates a notional implementation of CM within the framework of an HLA simulation federation. Software interfaces to HLA are not shown in the figure. Federations thus composed may be useful to the community because of their potential enhancement of nuclear power plant emergency preparedness plans as well their alignment with DoD interest in unmanned systems and the JTF-CS tasking to support civil authorities responding to CBRNE incidents and the training requirements associated with both (JTF-CS 2015b). Specific objectives, available resources, and security constraints of individual users are the major drivers in specific model selection.

For illustrative purposes, a commercial-off-the-shelf software package called STAGE from Presagis Modeling \& Simulation Suite 15 (Presagis 2015b) is validated for UAV and agent modeling and is already HLA compliant. STAGE is shown in Figure 5 providing the agent, scenario management, and data logging functionality identified as needed in Figure 4. Further using validated United States Geological Survey elevation, imagery, and feature data, Presagis Terra Vista generates the starting terrain and surface features while the Presagis Dynamic Terrain Server modifies the terrain in response to disruptive impulses such as an earthquake. Finally, illustrated in Figure 5, the HLA-compliant HeliSim (Presagis 2015a) models RUAS functionality.

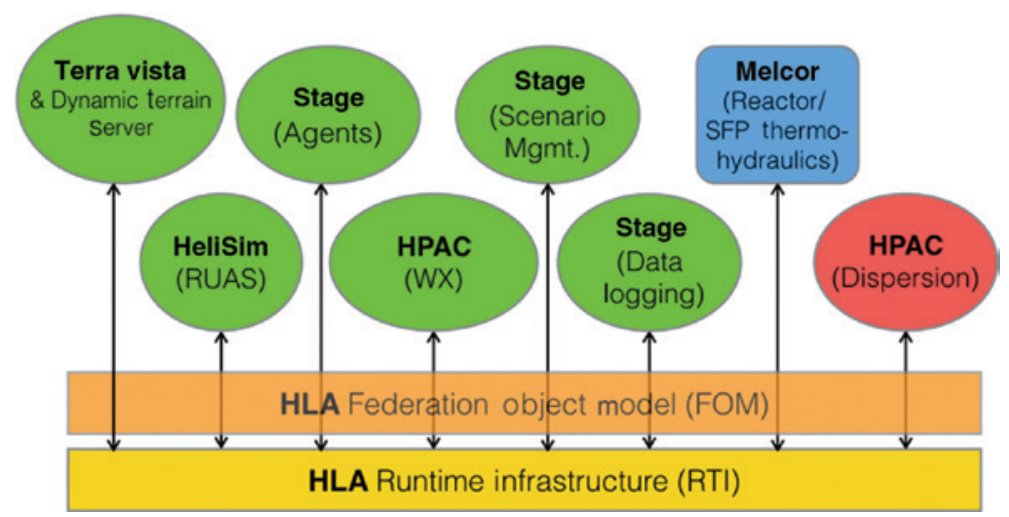

Figure 5: Notional LVC Simulation Framework as a HLA Federation. 
Continuing for illustrative purposes, high fidelity modeling of reactor thermohydraulics is a key component of the overall accuracy of RUAS water resupply to the SFP. Sandia National Lab's MELCOR model is extensively tested, verified, and widely use in the literature (Wang et al. 2005; Darnowski et al. 2015; Fernandez-Moguel 2015; Fernandez-Moguel and Birchley 2015; Polo-Labarrios and Espinosa-Paredes 2015; Sevón 2015; Wang et al. 2015a,b). Figure 5 indicates MELCOR providing Figure 4 Reactor/SFP functionality. MELCOR is not real-time and not HLA compliant. A software interface to HLA would be required and procedures to deal with non-real time data interoperating with real time data determined (McLean et al. 2004).

Illustrated in Figure 5, the Hazard Prediction and Assessment Capability (HPAC) provides radiation dispersion functionality. HPAC also includes an integrated weather model capable of ingesting real time data or pulling from historical databases (DoD 2013). Like MELCOR and confirming the current paradigm illustrated in Figure 2, HPAC is not HLA compliant (Lee 2002). So despite being able to run very fast analysis, HPAC requires additional software and procedures for integrating it into an HLA federation.

Analysis of existing RUAS likely to be adapted for SFP water delivery tasks is a logical follow-on. Output data from the SFP model is critical to RUAS operations and may provide a convenient set of response variables for RUAS analysis. Optimal system behavior is achieved when SFP water level and temperature are within normal operating range and in our case from (Collins and Hubbard 2001). These form the boundaries of our response variable. Initial conditions and inputs to each of the modules are treatment and noise variables. Standard experimental design practices can now be applied to map the capability envelope for a variety of RUAS platforms on a range of initial conditions and operating environments providing insight for optimal RUAS design or employment strategies. But as Hodson and Hill note, experimental design within a federation of simulations is not a trivial endeavor and very little work has been done in this area (Hodson and Hill 2014).

\section{Applications and Future Research}

Response to a nuclear disaster is a large, complex and time critical endeavor (Johnson 2006; TEPCO 2013; JTF-CS 2015a). Recent changes to the policies and regulations regarding emergency preparedness made by NRC (NRC 2011) and the establishment of a new regulatory body in Japan (Japan 2012) highlight the level of effort required to properly prepare for emergency response. The CM presented 
above may enable modeling, simulation and analysis of various unmanned system interventions into nuclear disaster emergency preparedness, response, and mitigation. For nuclear disasters that breach site defenses, unmanned systems solutions promise significant planning advantages over past expedient, high-risk, and marginally effective solutions. NRC also tests the validity of such plans through exercises and training on a regular basis (NRC 2014C). The discussion above highlights one potential RUAS intervention capability through a scenario actually experienced at Fukushima Dai-ichi. More importantly the RUAS example illustrates a SoS approach that is applicable to the entire spectrum of unmanned systems both present and in the future.

In terms of disaster response, the inherent extensibility of our SoS CM, like other M\&S approaches to incident management, offers several advantages including, but not limited to:

- Projecting incident impact

- Testing emergency response plans

- Training response personnel

- Cost savings over live exercises

- Strategy exploration/war-gaming

- Long range resource planning

- Force lay-down evaluation

(Jain and McLean 2006)

Additionally, constructing a LVC simulation framework from a CM using the SoS approach may require software interfaces for selected simulation but addresses incident management issues identified by McLean et al. that are applicable to the fore mentioned nuclear disaster scenarios.:

- Appropriate representation of the selected scenario

- Heterogeneous simulation integration

- Time synchronization

- Run-time execution control

- Support for large multi-agency exercises

- Heterogeneous data server access

- Reusability

(McLean et al. 2007)

Future areas for research include instantiating and analyzing an interacting suite of simulation models based on the proposed CM. The RUAS example above is but one of many possible unmanned system instantiations that may be considered. One major advantage of constructing analysis tools as systems of simulation systems is the inherent extensibility to other applications it provides. Modifying 
a given simulation for analysis of other types of unmanned systems performing the same or different tasks within the nuclear disaster response environment is relatively straightforward and significantly increases knowledge regarding the emergent behavior of unmanned systems in complex environments.

While complete simulation and federation composability has proven too lofty a goal (Kasputis and Ng 2000; Davis and Anderson 2004; Proctor and Gerber 2004), degrees of composability within simulations and federations are achievable (Tolk et al. 2007; Hodson and Hill 2014; Petty et al. 2014). Composable simulation systems are extensible to other applications and the logical extension of this work is training of nuclear response teams. Simulation is already being used in training these first responders (INNG 2015), but just as emergent behavior occurs within the simulation system, new emergent behaviors will occur when a new tool is introduced into the integrated response team. Large scale, fully integrated training scenarios give planners and leaders knowledge of how their entire team will respond when a new tool is introduced. The CM proposed here is a first step toward the integration of incident management simulations and response technology simulations called for by Jain and McLean: “The goal is to have a number of interoperable $M \& S$ tools available together with an ability to select and integrate the appropriate tools based on the situation at hand, and to create a simulated representation of the situation using data available in standard formats and structures" (Jain and McLean 2006). Further research is required to achieve this goal.

\section{References}

Adalja, Amesh A., Tara Kirk Sell, Sanjana J. Ravi, Katie Minton and Ryan Morhard (2015) "Emergency Preparedness in the 10-Mile Emergency Planning Zone Surrounding Nuclear Power Plants," Journal of Homeland Security and Emergency Management, 12(1):81-100.

Ai-Omari, M. A. R., M. A. Jaradat and M. Jarrah (2013) "Integrated Simulation Platform for Indoor Quadrotor Applications," 9th International Symposium on Mechatronics and its Applications (ISMA) 9-11 April 2013.

Alexis, K., G. Nikolakopoulos, A. Tzes and L Dritsas (2009) Coordination of Helicopter UAVs for Aerial Forest-Fire Surveillance, Applications of Intelligent Control to Engineering Systems. Springer.

Alver, Yucel, Murat Ozdogan and Enver Yucesan (2012) "Assessing the Robustness of UAV Assignments." 2012 Winter Simulation Conference (WSC), 1/1/2012.

ANS (2012) "Status of Spent Fuel in the Unit 1 Through 6 and Common Spent-Fuel Pools at the Fukushima Daiichi Nuclear Power Station.” In Fukushima Daiichi: ANS Committee Report, Appendix G. American Nuclear Society Special Committee on Fukushima.

Baker, Jean (2012) Capabilities Report 2012 West Desert Test Center. http://oai.dtic.mil/oai/ oai?\&verb=getRecord\&metadataPrefix=html\&identifier=ADA559993: Dugway Proving Ground. 
Bernard, Markus, Konstantin Kondak and Günter Hommel (2008) "A Slung Load Transportation System Based on Small Size Helicopters." In: Autonomous Systems-Self-Organization, Management, and Control. Springer, pp. 49-61.

Brantley, Mark W., Willie J. McFadden and Mark J. Davis (2002) "Expanding the Trade Space: An Analysis of Requirements Tradeoffs Affecting System Design. (Tutorial).” Acquisition Review Quarterly, (1):1.

Campbell, S., M. Abu-Tair and W. Naeem (2014) "An Automatic COLREGS-Compliant Obstacle Avoidance System for an Unmanned Surface Vehicle," Proceedings of the Institution of Mechanical Engineers Part M-Journal of Engineering for the Maritime Environment, 228(2):108-121.

Çelik, Turgay, Göktuğ F. Gökdoğan, Karahan Öztürk and Bircan Sarikaya (2013) “An HLA-Based Tactical Environment Application Framework," The Journal of Defense Modeling and Simulation: Applications, Methodology, Technology, 10(3):217-233.

Chaimatanan, Supatcha, Daniel Delahaye and Marcel Mongeau (2013) "Strategic Deconfliction of Aircraft Trajectories." 2nd International Conference on Interdisciplinary Science for Innovative Air Traffic Management, Toulouse, France, July 2013.

Chang, Joseph C., Steven R. Hanna, Zafer Boybeyi and Pasquale Franzese (2005) “Use of Salt Lake City URBAN 2000 Field Data to Evaluate the Urban Hazard Prediction Assessment Capability (HPAC) Dispersion Model," Journal of Applied Meteorology, 44(4):485-501.

Collins, T. E. and G. Hubbard (2001) Technical Study of Spent Fuel Pool Accident Risk at Decommissioning Nuclear Power Plants. In NUREG-1738: Nuclear Regulatory Commission.

Covelli, Javier M., Jannick P. Rolland, Michael Proctor, J. Peter Kincaid and P. A. Hancock (2010) "Field of View Effects on Pilot Performance in Flight," The International Journal of Aviation Psychology, 20(2):197-219.

Darnowski, Piotr, Eleonora Skrzypek, Piotr Mazgaj, Konrad Świrski and Pascal Gandrille (2015) “Total Loss of AC Power Analysis for EPR Reactor,” Nuclear Engineering \& Design, 289:8-18.

Davis, Paul K. and Robert H. Anderson (2004) "Improving the Composability of DoD Models and Simulations," Journal of Defense Modeling \& Simulation, 1(1):5-17.

Davis, Paul K. and A. Tolk (2007) "Observations on New Developments in Composability and Multi-Resolution Modeling." Winter Simulation Conference, 9-12 Dec. 2007.

Deverell, E (2012) "Investigating the Roots of Crisis Management Studies and Outlining Future Trajectories for the Field," Journal of Homeland Security and Emergency Management, 9(1):2.

DHS (2015) National Preparedness Goal. http://www.fema.gov/media-librarydata/1443799615171-2aae90be55041740f97e8532fc680d40/National_Preparedness_ Goal_2nd_Edition.pdf: Department of Homeland Security.

DoD (2013) “DOD 3150.08-M “Nuclear Weapon Accident Response Procedures” (NARP) Internet Supplement." Department of Defense. http://www.acq.osd.mil/ncbdp/narp/Radiation_ Data/Specialized_Radiological.htm.

DOE (2015) “Energy Technology Engineering Center Closure Project: The SRE Accident.” Accessed 3 June 2015. http://etec.energy.gov/Operations/Major_Operations/SRE_Accident.html.

Ellis, Christopher, Pavel Babenko and Brian Goldiez (2010) "Dynamic Terrain for Multiuser RealTime Environments," IEEE Computer Graphics \& Applications, 30(1):80-84.

EPA (2011) "Radiation Monitors Continue to Confirm That No Radiation Levels of Concern Have Reached the United States." [Press Release]. EPA. http://yosemite.epa.gov/opa/admpress.nsf/d0cf6618525a9efb85257359003fb69d/3724de8571e1b03f8525785c00041a7a \%210penDocument. 
FEMA (2014) Response Federal Interagency Operational Plan. https://www.fema.gov/medialibrary-data/1406719953589-4ab5bfa40fe82879611d945dd60230c4/Response_FIOP_ FINAL_20140729.pdf: Department of Homeland Security.

Fernandez-Moguel, L. and J. Birchley (2015) "Analysis of the Accident in the Fukushima Daiichi Nuclear Power Station Unit 3 with MELCOR_2.1,” Annals of Nuclear Energy, 83:193-215.

Fernandez-Moguel, Leticia (2015) “Comparative Assessment of PSI Air Oxidation Model Implementation in SCDAPSim3.5, MELCOR 1.8.6 and MELCOR 2.1," Annals of Nuclear Energy, 81:134-142.

Fisher, David A (2006) An Emergent Perspective on Interoperation in Systems of Systems. http://oai.dtic.mil/oai/oai?\&verb=getRecord\&metadataPrefix=html\&identifier= ADA449020: Carnegie Mellon Software Engineering Institute.

Flint, M., E. Fernandez and W. D. Kelton (2009) "Simulation Analysis for UAV Search Algorithm Design Using Approximate Dynamic Programming,” Military Operations Research, 14(2):41-50.

Gaffney, Helen and Alasdair Vincent (2011) “Modeling Information Operations in a Tactical-level Stabilization Environment," Journal of Defense Modeling \& Simulation, 8(2):105.

Girault, N., L. Bosland and J. Dienstbier (2010) "LWR Severe Accident Simulation Fission Product Behavior in FPT2 Experiment," Nuclear Technology, 169(3):218-238.

Graniela, Benito and Michael D. Proctor (2012) “A Network-Centric Terrain Database Regeneration Architecture," The Journal of Defense Modeling and Simulation: Applications, Methodology, Technology, 10(3):247-261.

Green, Chris, Bruce C. Leibrecht, Jeffrey E. Fite (2011) After Action Review Guide for Trainers of Virtual Battlespace-2 Missions. http://oai.dtic.mil/oai/oai?\&verb=getRecord\&metadataPr efix $=$ html\&identifier=ADA548308: United States Army Research Institute for the Behavioral and Social Sciences.

Hamilton, John A. (2006) "A Conceptual Model for Interoperable Command and Control Acquisition," Journal of Defense Modeling \& Simulation, 3(2):125-138.

Hanna, Steven and Joseph Chang (2015) "Skyscraper Rooftop Tracer Concentration Observations in Manhattan and Comparisons with Urban Dispersion Models," Atmospheric Environment, 106(2015):215-222.

Heffelfinger, David G., Craig M. Tuckett and Jeffrey R. Ryan (2013) “The Military’s Response to Domestic CBRNE Incidents," Journal of Homeland Security and Emergency Management, 10(1):57-75.

Hill, Alexander (2003) Using the Hazard Prediction and Assessment Capability (HPAC) Hazard Assessment Program for Radiological Scenarios Relevant to the Australian Defence Force. http://oai.dtic.mil/oai/oai?\&verb=getRecord\&metadataPrefix=html\&identifier= ADA416823: DSTO Platforms Sciences Laboratory.

Hodson, Douglas D. and Raymond R. Hill (2014) "The Art and Science of Live, Virtual, and Constructive Simulation for Test and Analysis," Journal of Defense Modeling \& Simulation, 11(2):77-89.

Hodson, Douglas D., Bruce L. Esken, Alex J. Gutman and Raymond R. Hill (2014)

"Quantifying Radar Measurement Errors in a Live-Virtual-Constructive Environment to Determine System Viability: A Case Study," Journal of Defense Modeling \& Simulation, 11(2):115-124.

Holden, Trevor and Charles Dickerson (2013) “A ROSETTA Framework for Live/Synthetic Aviation Tradeoffs: Preliminary Report," Proceedings of the 2013 8th International Conference on System of Systems Engineering, 218-223. doi: 10.1109/SYSoSE.2013.6575270. 
Hollenbach, James W. and William L. Alexander (1997) "Executing the DoD Modeling and Simulation Strategy - Making Simulation Systems of Systems a Reality," Proceedings of the 29th Winter Simulation Conference, 948-954. doi: 10.1145/268437.268690.

Hsu, Jeremy (11/2014) “The Weight Of War,” Popular Science, 285(5):60-65.

Hu, Liang, Yapei Zhang, Longze Li, G. H. Su, Wenxi Tian and Suizheng Qiu (2015). "Investigation of Severe Accident Scenario of PWR Response to LOCA Along with SBO," Progress in Nuclear Energy, 83(2015):159-166.

lanovsky, Edward and Joseph Kreimer (2011) "An Optimal Routing Policy for Unmanned Aerial Vehicles (Analytical and Cross-Entropy Simulation Approach)," Annals of Operations Research, 189(1):215-253.

IEEE (2010) IEEE Standard for Modeling and Simulation (MI\&S) High Level Architecture (HLA)Framework and Rules. In IEEE Std 1516-2010 (Revision of IEEE Std 1516-2000). doi: 10.1109/ IEEESTD.2010.5553440.

IEEE (2012) IEEE Standard for Distributed Interactive Simulation-Application Protocols. In IEEE Std 1278.1-2012 (Revision of IEEE Std 1278.1-1995). doi: 10.1109/IEEESTD.2012.6387564.

INNG (2015) Vibrant Response Q and A. http://www.atterburymuscatatuck.in.ng.mil/Portals/18/PageContents/Training/JE/Q_and_A.pdf: Indiana Army National Guard.

Jain, Sanjay and Charles R. McLean (2006) "An Integrating Framework for Modeling and Simulation for Incident Management," Journal of Homeland Security and Emergency Management, 3(1):1-5.

Japan, National Diet of (2012) Act for Establishment of the Nuclear Regulation Authority. In Act No. 47, edited by National Diet of Japan. https://www.nsr.go.jp/data/000067231.pdf: Organisation for Economic Cooperation \& Development.

Johnson, Thomas (2006) The Battle of Chernobyl. http://icarusfilms.com/new2007/batt.html: Icarus Fllms. Film.

Jones, Michael C. (2015) “Composability.” In: (Larry B. Rainey and Andreas Tolk, eds.) Modeling and Simulation Support for System of Systems Engineering Applications. Hoboken, New Jersey: Wiley, pp. 45-73.

JTF-CS (2015a) JTF-CS 101 Brief v5.5. edited by Department of Defense. http://www.jtfcs. northcom.mil/Documents/JTF-CS 101 Brief v5.5 (28 APR 2015).pdf: Joint Task Force Civil Support.

JTF-CS (2015b) “JTFCS Operational Focus.” US Northern Command Accessed 30 April. http:// www.jtfcs.northcom.mil/OperationalFocus.aspx.

Kasputis, S. and H. C. Ng (2000) "Composable Simulations," Proceedings of the 2000 Winter Simulation Conference, 2:1577-1584.

Law, Averill M. and W. David Kelton (2000) Simulation Modeling and Analysis. 3rd ed, McGrawHill Series in Industrial Engineering and Management Science. Boston: McGraw-Hill. Bibliographies; Non-fiction.

Lee, Ronald W. (2002) Moving the Hazard Prediction and Assessment Capability to a Distributed, Portable Architecture. ORNL Oak Ridge National Laboratory (US). doi: $10.2172 / 814542$.

Liang, Wei, S. N. Lam Nina, Xiaojun Qin and Wenxue Ju (2015) “A Two-Level Agent-Based Model for Hurricane Evacuation in New Orleans," Journal of Homeland Security and Emergency Management, 12(2):407-435.

Liu, X. F., Z. W. Guan, Y. Q. Song, and D. S. Chen (2014) “An Optimization Model of UAV Route Planning for Road Segment Surveillance," Journal of Central South University, 21(6):2501-2510. 
MacFarlane, J. W., O. D. Payton, A. C. Keatley, G. P. T. Scott, H. Pullin, R. A. Crane, M. Smilion, I. Popescu, V. Curlea and T. B. Scott (2014) “Lightweight Aerial Vehicles for Monitoring, Assessment and Mapping of Radiation Anomalies," Journal of Environmental Radioactivity, 136(2014):127-130.

Maier, Mark W. (1998) “Architecting Principles for Systems-of-Systems," Systems Engineering, 1(4):267-284.

Marconi, L., C. Melchiorri, M. Beetz, D. Pangercic, R. Siegwart, S. Leutenegger, R. Carloni, S. Stramigioli, H. Bruyninckx, P. Doherty, A. Kleiner, V. Lippiello, A. Finzi, B. Siciliano, A. Sala and N. Tomatis (2012) "The SHERPA Project: Smart Collaboration Between Humans and Ground-Aerial Robots for Improving Rescuing Activities in Alpine Environments," 2012 IEEE International Symposium on Safety, Security, and Rescue Robotics (SSRR):1-4. doi: 10.1109/SSRR.2012.6523905.

Mase, Kenichi (2013) “Wide-Area Disaster Surveillance Using Electric Vehicles and Helicopters," 2013 IEEE 24th International Symposium on Personal Indoor and Mobile Radio Communications (PIMRC):3466-3471. doi: 10.1109/PIMRC.2013.6666748.

Mase, Kenichi (2015) "Wide Area Surveillance Using Electric Vehicles and Limited-Flying-Time Helicopters," Sensors \& Transducers, 185(2):84-92.

McGonigle, A. J. S., A. Aiuppa, G. Giudice, G. Tamburello, A. J. Hodson and S. Gurrieri (2008) “Unmanned Aerial Vehicle Measurements of Volcanic Carbon Dioxide Fluxes," Geophysical Research Letters, 35(6):1-4.

McLean, Thom, Richard Fujimoto and Brad Fitzgibbons (2004) "Middleware for RealTime Distributed Simulations," Concurrency \& Computation: Practice \& Experience, 16(15):1483-1501.

McLean, Charles R, Sanjay Jain, Y Tina Lee and Guodong Shao (2007) An Integrated Simulation Environment For Incident Management Training. http://www.nist.gov/manuscript-publication-search.cfm?pub_id=822742: National Institute of Standards and Technology.

Meliza, Larry L., Stephen L. Goldberg, Donald R. Lampton (2007) After Action Review in Simulation-Based Training. http://www.dtic.mil/cgi-bin/GetTRDoc?AD=ADA474305: U.S. Army Research Institute for the Behavioral and Social Sciences.

Miller, C., A. Cubbage, D. Dorman, J. Grobe, G. Holahan, N. Sanfilippo (2011) Recommendations for Enhancing Reactor Safety in the 21st Century. http://pbadupws.nrc.gov/docs/ML1118/ ML111861807.pdf: Nuclear Regulatory Commission.

Morris, Jeffrey D., Michael R. Grimaila, Douglas D. Hodson, Colin V. McLaughlin and David R. Jacques (2014) "Using the Discrete Event System Specification to Model Quantum Key Distribution System Components," The Journal of Defense Modeling and Simulation: Applications, Methodology, Technology, 12(4):457-480.

NAWCTSD. 2014. NAWCTSD Research Project Summaries: Fiscal Years 2014-2015. http://www. navair.navy.mil/nawctsd/Programs/Files/NAWCTSD-FY-14-15-Grey-Book.pdf: USN.

NRC (2011) Criteria for Preparation and Evaluation of Radiological Emergency Response Plans and Preparedness in Support of Nuclear Power Plants: Guidance for Protective Action Strategies. http://purl.fdlp.gov/GPO/gpo54473: Washington, DC: U.S. Nuclear Regulatory Commission, FEMA, [2011] Rev. 1, Supplement 3. Technical reports; Non-fiction; Electronic document.

NRC (2012) Order Modifying Licenses with Regard to Reliable Spent Fuel Pool Instrumentation. In EA-12-051, edited by Nuclear Regulatory Commission. http://www.nrc.gov/docs/ ML1205/ML12056A044.pdf. 
NRC (2014a) 2002 Davis-Besse Reactor Pressure Vessel Head Degradation Knowledge Management Digest. http://www.nrc.gov/docs/ML1403/ML14038A119.pdf: Nuclear Regulatory Commission.

NRC (2014b) The Browns Ferry Nuclear Plant Fire of 1975 Knowledge Management Digest. http:// www.nrc.gov/docs/ML1321/ML13210A179.pdf: Nuclear Regulatory Commission.

NRC (2014c) NUREG-1350. edited by Nuclear Regulatory Commission. http://www.nrc.gov/ reading-rm/doc-collections/nuregs/staff/sr1350/v26/sr1350v26.pdf: NRC.

NRC (2015a) “Computer Codes.” Accessed 24 June 2015. http://www.nrc.gov/about-nrc/regulatory/research/safetycodes.html - sac.

NRC (2015b) “Operating Reactors.” Nuclear Regulatory Comission, Last Modified 18 Feb, 2015 Accessed 10 April 2015. http://www.nrc.gov/reactors/operating.html.

Oikawa, Kiyoshi (2015) Robot Technology for Nuclear Decommissioning of Fukushima Daiichi NPS. http://irid.or.jp/_pdf/20150421_1.pdf: International Research Institute for Nuclear Decommissioning (IRID).

OPA (2013a) “Chernobyl Nuclear Power Plant Accident.” Nuclear Regulatory Commission Accessed 24 June 2015. http://www.nrc.gov/reading-rm/doc-collections/fact-sheets/ chernobyl-bg.html.

OPA (2013b) “Three Mile Island Accident.” Nuclear Regulatory Commission Accessed 10 April 2015. http://www.nrc.gov/reading-rm/doc-collections/fact-sheets/3mile-isle.html.

Ouyang, Jian, Yi Zhuang, Min Lin and Jia Liu (2014) "Optimization of Beamforming and Path Planning for UAV-Assisted Wireless Relay Networks," Chinese Journal of Aeronautics, 27(2):313-320.

Özhan, Gürkan, Halit Oğuztüzün, and Pinar Evrensel (2008) “Modeling of Field Artillery Tasks with Live Sequence Charts." Journal of Defense Modeling \& Simulation, 5(4):219-252.

PB Farradyne, a District of PBQD. 2005. Use of Unmanned Aerial Vehicles in Traffic Surveillance and Traffic Management. http://www.i95coalition.org/wp-content/uploads/2015/03/ Report_TechMemo_UAV_FL.pdf: Technical Memorandum Prepared for: Florida Department of Transportation.

Peräjärvi, K., J. Lehtinen, R. Pöllänen and H. Toivonen (2008) “Design of an Air Sampler for a Small Unmanned Aerial Vehicle," Radiation Protection Dosimetry, 132(3):328-333.

Perhinschi, M. G., M. R. Napolitano and S. Tamayo (2010) “Integrated Simulation Environment for Unmanned Autonomous Systems-Towards a Conceptual Framework," Modelling \& Simulation in Engineering, 2010:1-12.

Petty, Mikel D., Jungyoon Kim, Salvador E. Barbosa and Jai-Jeong Pyun (2014) "Software Frameworks for Model Composition," Modelling and Simulation in Engineering, 2014:1-18.

Platt, Nathan, William Ross Kimball and Jeffry T. Urban (2014) “The Use of Probabilistic Plume Predictions for the Consequence Assessment of Atmospheric Releases of Hazardous Materials," International Journal of Environment and Pollution, 55:1-5.

Polo-Labarrios, M. A. and G. Espinosa-Paredes (2015) "Comparative Study of the Hydrogen Generation During Short Term Station Blackout (STSBO) in a BWR," Annals of Nuclear Energy, 83(2015):274-282.

Presagis (2015a) “Helisim.” Presagis Accessed 2 July 2015. http://www.presagis.com/products_services/products/modeling-simulation/simulation/helisim/.

Presagis (2015b) “M\&S Suite.” Presagis Accessed 2 July 2015. http://www.presagis.com/products_services/products/modeling-simulation/.

Pripyat.com (2006) “Chernobyl Disaster: Helicopter crash near Nuclear Power Plant.” [video]. https://youtu.be/8s40uKLCjcU. 
Proctor, Michael D. and G. Paulo (1996) “Modeling in Support of Operational Testing," Mathematical and Computer Modelling, 23(1-2):9-14.

Proctor, M. D. and P. E. Connors (2000) "Data Representation for Sensor Models Within a Synthetic Natural Environment (SEDRIS)," Transactions of the Society for Computer Simulation International, 17(2):46-53.

Proctor, Michael D. and William J. Gerber (2004) “Line-of-Sight Attributes for a Generalized Application Program Interface," Journal of Defense Modeling \& Simulation, 1(1):43-57.

Proctor, Michael D., Amy Posey-Macalintal and Dennis Kulonda (2003) "Why the'T' in SMART: A Constructive Synergy,” Acquisition Review Quarterly, 10(3):284-299.

Proctor, Michael D., Michael Panko and Sharlene J. Donovan (2004) “Considerations for Training Team Situation Awareness and Task Performance Through PC-Gamer Simulated Multiship Helicopter Operations," International Journal of Aviation Psychology, 14(2):191-205.

Proctor, Michael D., Maria Bauer and Thomas Lucario (2007) "Helicopter Flight Training Through Serious Aviation Gaming," Journal of Defense Modeling \& Simulation, 4(3):277-294.

Rami, Nabil and Michael D. Proctor (2007) "Real Time Physically-Based Modeling and Simulation of Cratering and Fragmentation of Terrain," Simulation-Transactions of the Society for Modeling and Simulation International, 83(12):830-842.

Robinson, S (2008a) “Conceptual Modelling for Simulation Part I: Definition and Requirements," Journal of the Operational Research Society, 59(3):278-290.

Robinson, S. (2008b) "Conceptual Modelling for Simulation Part II: A Framework for Conceptual Modelling," Journal of the Operational Research Society, 59(3):291-304.

Robinson, Stewart (2010) Conceptual Modeling for Discrete-Event Simulation. Boca Raton: CRC Press. Bibliographies; Non-fiction; Electronic document.

Saggiani, G. M. and B. Teodorani (2004) "Rotary Wing UAV Potential Applications: An Analytical Study Through a Matrix Method," Aircraft Engineering and Aerospace Technology, 76(1):6-14.

Sawyer, Taylor L. and Shad Deering (2013) “Adaptation of the US Army's After-Action Review for Simulation Debriefing in Healthcare," Simulation in Healthcare-Journal of the Society for Simulation in Healthcare, 8(6):388-397.

Sevón, Tuomo (2015) “A MELCOR Model of Fukushima Daiichi Unit 1 Accident," Annals of Nuclear Energy, 85 (2015):1-11.

Sharma, Sanjay K., Robert Sutton, Amit Motwani and Andy Annamalai (2014) “Non-Linear Control Algorithms for an Unmanned Surface Vehicle," Proceedings of the Institution of Mechanical Engineers Part M-Journal of Engineering for the Maritime Environment, 228(2):146-155.

Sheng, Li, ZhenXin Song, JiangKai Hu, Kai Lü, Hua Tong, Bing Li and QingDang Qiao (2015) "The Comparison of Ensemble or Deterministic Dispersion Modeling on Global Dispersion During Fukushima Dai-ichi Nuclear Accident," Science China Earth Sciences, 58(3):356-370.

Singh, Ashish, Scott N. Spak, Elizabeth A. Stone, Jared Downard, Robert L. Bullard, Mark Pooley, Pamela A. Kostle, Matthew W. Mainprize, Michael D. Wichman, Thomas M. Peters, Douglas Beardsley and Charles O. Stanier (2015) "Uncontrolled Combustion of Shredded Tires in a Landfill - Part 2: Population Exposure, Public Health Response, and an Air Quality Index for Urban Fires," Atmospheric Environment, 104(2015):273-283.

Sokolowski, John A., Catherine M. Banks and Brent Morrow (2012) "Using an Agent-Based Model to Explore Troop Surge Strategy," Journal of Defense Modeling \& Simulation, 9(2):173-186. 
Stephens, Keri K., Ehsan Jafari, Stephen Boyles, L. Ford Jessica and Yaguang Zhu (2015) "Increasing Evacuation Communication Through ICTs: An Agent-Based Model Demonstrating Evacuation Practices and the Resulting Traffic Congestion in the Rush to the Road," Journal of Homeland Security and Emergency Management, 12(3):497-528.

Sugiyama, Gayle (2011) Modeling and Simulation of Hazardous Material Releases for Homeland Security Applications. http://www.nist.gov/el/msid/upload/Hazardous_Material. pdf: DHS.

Svec, Petr, Atul Thakur, Erie Raboin, Brual C. Shah and Satyandra K. Gupta (2014) “Target Following with Motion Prediction for Unmanned Surface Vehicle Operating in Cluttered Environments," Autonomous Robots, 36(4):383-405.

TEPCO (2013) The Development of and Lessons from the Fukushima Daiichi Nuclear Accident. http://www.tepco.co.jp/en/decommision/accident/images/outline01.pdf: Tokyo Electric Power Company.

Tolk, Andreas, Saikou Diallo and Charles Turnitsa (2007) “Applying the Levels of Conceptual Interoperability Model in Support of Integratability, Interoperability, and Composability for System-of-Systems Engineering," Journal of Systemics, Cybernetics and Informatics, 5(5):65-74.

Tolk, Andreas and James A. Muguira (2003) "The Levels of Conceptual Interoperability Model," Proceedings of the 2003 Fall Simulation Interoperability Workshop, 7:1-11.

Tomaszewski, Brian, Michael Judex, Joerg Szarzynski, Christine Radestock and Lars Wirkus (2015) “Geographic Information Systems for Disaster Response: A Review," Journal of Homeland Security and Emergency Management, 12(3):571-602.

Towler, Jerry, Bryan Krawiec and Kevin Kochersberger (2012) "Radiation Mapping in Post-Disaster Environments Using an Autonomous Helicopter," Remote Sensing 4(7):1995-2015.

TRMC (2015) “TENA Introductory Material.” TRMC Accessed 1 July 2015. https://www.tena-sda. org/display/intro/Documentation.

Ünal, Ömer and Okan Topçu (2014) “Modelling Unmanned Surface Vehicle Patrol Mission with Coalition Battle Management Language (C-BML)." Journal of Defense Modeling \& Simulation, 11(3):277-308.

UNSCEAR (2000) “Annex J: Exposures and Effects of the Chernobyl Accident." In UNSCEAR assessments of the Chernobyl accident. http://www.unscear.org/docs/reports/2000/ Volumell_Effects/Annex)_pages451-566.pdf: United Nations Scientific Committee on the Effects of Atomic Radiation.

UNSCEAR (2014) Levels and Effects of Radiation Exposure Due to the Nuclear Accident After the 2011 Great East-Japan Earthquake and Tsunami. In Sources, Effects and Risks of lonizing Radiation. Report to the General Assembly with Scientific Annexes. http://www.unscear. org/docs/publications/2013/UNSCEAR_2013_Report_Vol.I.pdf: United Nations Scientific Committee on the Effects of Atomic Radiation.

USGS (2016) “Earth Explorer.” Dept. of the Interior. http://earthexplorer.usgs.gov/.

UTM (2007) “Palm Bay, FL, Deploys Unmanned Air Vehicles.” The Urban Transportation Monitor, 2 Feb 2007, 1, 1. https://trid.trb.org/view.aspx?id=808168.

van Blyenburgh, Peter (2000) UAVs - Current Situation and Considerations for the Way Forward. http://oai.dtic.mil/oai/oai?\&verb=getRecord\&metadataPrefix=html\&identifier= ADP010752: Defense Technical Information Center.

Van Niekerk, Dewald, Christo Coetzee, Doret Botha, John Murphree Michael, Kristel Fourie, Tanya Le Roux, Gideon Wentink, Leandri Kruger, Lesego Shoroma, Kylah Genade, Suna Meyer and E. Annandale (2015) "Planning and Executing Scenario Based Simulation 
Exercises: Methodological Lessons," Journal of Homeland Security and Emergency Management, 12(1):193-210.

Wang, Te-Chuan, Shih-Jen Wang and Jyh-Tong Teng (2005) "Comparison of Severe Accident Results Among SCDAP/RELAP5, MAAP, and MELCOR Codes," Nuclear Technology, 150(2):145-152.

Wang, Jun, Michael L. Corradini, Wen Fu, Troy Haskin, Yapei Zhang, Wenxi Tian, Guanghui Su and Suizheng Qiu (2015a) "Simulation of the PHEBUS FPT-1 Experiment Using MELCOR and Exploration of the Primary Core Degradation Mechanism," Annals of Nuclear Energy, 85(2015):193-204.

Wang, Jun, Yapei Zhang, Keyou Mao, Yujia Huang, Wenxi Tian, Guanghui Su and Suizheng Qiu (2015b) “MELCOR Simulation of Core Thermal Response During a Station Blackout Initiated Severe Accident in China Pressurized Reactor (CPR1000)." Progress in Nuclear Energy, 81 (2015):6-15.

WNA (2012) “Fukushima: Background on Fuel Pools.” World Nuclear Association, Last Modified Sep. 2012. http://www.world-nuclear.org/info/Safety-and-Security/Safety-of-Plants/ Appendices/Fukushima-Fuel-Ponds-Background/.

WNA (2015) “Nuclear Database.” World Nuclear Association Accessed 10 April. http://www. world-nuclear.org/NuclearDatabase/rdResults.aspx?id=27569.

WSC (2012) WSC Brings Severe Accident Modeling to 3KEYMASTER With MELCOR. https://www. ws-corp.com/wsc08/wsc14/LiveEditor/images/newsletter/2012/index.html: Western Services Corporation.

Yilmaz, Levent (2004) “On the Need for Contextualized Introspective Models to Improve Reuse and Composability of Defense Simulations," Journal of Defense Modeling \& Simulation, 1(3):141-151.

Zeigler, Bernard P., Tag Gon Kim and Herbert Praehofer (2000) Theory of Modeling and Simulation: Integrating Discrete Event and Continuous Complex Dynamic Systems. 2nd ed. San Diego, [Calif.]; London: Academic Press. Bibliographies; Non-fiction.

Zittel, Randy C. (2001) "The Reality of Simulation-Based Acquisition - And an Example of U.S. Military Implementation,” Acquisition Review Quarterly, 2001(2):121-132. 